\title{
IncRNAs: function and mechanism in cartilage development, degeneration, and regeneration
}

\author{
Jian Zhu ${ }^{1,2+}$, Wei Yu ${ }^{1,2 \dagger}$, Yitian Wang ${ }^{1,2 \dagger}$, Kaishun Xia ${ }^{1,2}$, Yuluan Huang ${ }^{3}$, Ankai Xu ${ }^{1,2}$, Qixin Chen ${ }^{1,2}$, Bing Liu ${ }^{1,2}$, \\ Huimin TaO ${ }^{1,2+}$, Fangcai $\mathrm{Li}^{1,2+}$ and Chengzhen Liang ${ }^{1,2^{*}+}$ (D)
}

\begin{abstract}
With the increasing incidence of cartilage-related diseases such as osteoarthritis $(\mathrm{OA})$ and intervertebral disc degeneration (IDD), heavier financial and social burdens need to be faced. Unfortunately, there is no satisfactory clinical method to target the pathophysiology of cartilage-related diseases. Many gene expressions, signaling pathways, and biomechanical dysregulations were involved in cartilage development, degeneration, and regeneration. However, the underlying mechanism was not clearly understood. Recently, lots of long non-coding RNAs (IncRNAs) were identified in the biological processes, including cartilage development, degeneration, and regeneration. It is clear that IncRNAs were important in regulating gene expression and maintaining chondrocyte phenotypes and homeostasis. In this review, we summarize the recent researches studying IncRNAs' expression and function in cartilage development, degeneration, and regeneration and illustrate the potential mechanism of how they act in the pathologic process. With continued efforts, regulating IncRNA expression in the cartilage regeneration may be a promising biological treatment approach.
\end{abstract}

Keywords: LncRNA, Cartilage, Arthritis, IDD, Mesenchymal stem cell, Differentiation

\section{Introduction}

Nowadays, the disease owing to cartilage widely influences people's lives, especially in aging society countries. Cartilage diseases such as osteoarthritis (OA) and intervertebral disc degeneration (IDD) will cause pain and movement limitations [1]. The main cause of OA and IDD is the progressive destruction of cartilage [2, 3]. In order to cure osteoarthritis and intervertebral disc degeneration, it is necessary to understand the cartilage's development, degeneration, and regeneration.

LncRNAs attract more and more attention owing to their abundance functions in various tissues [4]. LncRNAs

\footnotetext{
* Correspondence: liangchengzhen@zju.edu.cn; lifangcai@zju.edu.cn; huimintao@zju.edu.cn

†Jian Zhu, Wei Y, and Yitian Wang are co-first authors.

Huimin Tao, Fangcai Li, and Chengzhen Liang are co-communication authors.

${ }^{1}$ Department of Orthopedics, 2nd Affiliated Hospital, School of Medicine, Zhejiang University, \#88 Jie Fang Road, Hangzhou 310009, Zhejiang, People's Republic of China

${ }^{2}$ Orthopedics Research Institute of Zhejiang University, \#88, Jiefang Road, Hangzhou 310009, China

Full list of author information is available at the end of the article
}

are virtually transcribed by RNA polymerase II and contain RNA-processing signals such as poly (A) tails and 5' caps [5]. Owing to the lack of open reading frame, lncRNAs were thought of as "junk RNAs." On the progress in the research, lncRNAs were found to act a crucial role in the biological process. LncRNAs are alternatively spliced and undergo a process to remove the intronic sequence [6]. LncRNAs are about 200 nucleotides to $100 \mathrm{~kb}$, similar in the structure of mRNA transcripts but without encoding a protein function [7]. According to the location relative to the gene locus, IncRNAs can be divided into five categories: sense, antisense, bidirectional, intronic, and intergenic [8]. LncRNAs are more species-specific and less conserved than the protein-encoding genes. LncRNAs can act as a regulator in various biological processes such as tumor development, stem cell differentiation, epigenetic regulation, immune response, and inflammation-related diseases [9-13]. LncRNAs can act as an indicator, biomarker, and therapy target in the physiologic and pathologic processes, including cartilage development, degeneration, and regeneration [14-16]. 
Now in this report, we will illustrate the role of lncRNAs in cartilage development, degeneration, and regeneration $[14,17]$ (Table 1). Hopefully, the brief introduction could afford a deep understanding of chondrocyte degeneration and a new target to cure cartilage degeneration.

\section{The role of IncRNAs in cartilage development}

Cartilage could be divided into three types: hyaline, elastic, and fibrocartilage [33]. Cartilage was thought of as a simple structure, because it contains only one type of cell and its extracellular matrix (ECM) contains only three components: water, collagen, and proteoglycan [34]. Chondrogenesis is the primary process in cartilage development [35]. Cartilage development contains five stages [36]: the initial stage mesenchymal stem cell, commitment into chondrocyte, chondrocyte differentiation, chondrocyte hypertrophy, and calcification and degradation of cartilage matrices (Fig. 1).

In the initial stage of cartilage formation, mesenchyme cells begin to condense. The BMP-SMAD4 signaling pathway plays a crucial role in mesenchymal condensation [37]. BMPR1B is a principal receptor for BMPs and GDF5. It will induce the skeletal element hypoplasia for the lack of BMPR1B. LncRNA HIT could regulate the BMP-SMAD4 signaling pathway through two mechanisms [38]. Firstly, LncRNA HIT binds to its associated protein such as p100 and CBP to regulate the expression of Bmprlb. Secondly, lncRNA HIT locates within the HOXA locus [39]. LncRNA HIT could regulate the expression of Hoxa13 and Hoxa11. Meantime, Hoxa13 regulates Bmp2 and Bmp7, and Hoxa11 regulates Runx2 in the BMP signaling pathway. Runx2 was reported to be expressed in cartilage condensation in the cartilage anlagen of the forelimb zeugopod [40]. Therefore, lncRNA HIT plays a major role in the initial stage of cartilage formation.

Sex-determining region Y (SRY)-box 9 (Sox9) plays an important role in promoting mesenchymal stem cells to the stage of commitment into chondrocytes [41]. Sox9 determines cell fate in cells derived from all three germ layers. It plays an important role in the initial stage of cartilage development. Mutation of Sox9 will disrupt the cartilage formation to cause campomelic dysplasia [36]. Aryl hydrocarbon receptor (AHR) is a conserved receptor from invertebrates to vertebrates, loss of which will protect against toxicity phenotypes, including cardiac malformation, cartilage malformation, and reduced peripheral blood flow [42, 43]. Garcia et al. [44] found that a novel lncRNA named slincR is associated with AHR2 and sox9b expression during normal development. LncRNA slincR acts as an intermediate between AHR2 and sox9b mRNA. However, more researches are needed to illustrate the mechanism of that reduction of sox $9 \mathrm{~b}$ caused by LncRNA slincR. Another lncRNA termed lncRNA ROCR was located $94 \mathrm{~kb}$ upstream of the location of SOX9. LncRNA is prone to regulate the expression of the gene nearby [45]. LncRNA ROCR aggregates more in the cytoplasm than in the nucleus, suggesting that it may regulate the expression of SOX9 in an indirect way. RNAi and LNA GapmeR approach were used to identify the effect of IncRNA ROCR on the expression of SOX9 and chondrogenesis [46]. The study showed that IncRNA ROCR contributes to the expression of SOX9, and lncRNA ROCR is necessary for matrix

Table 1 Functional characterization of IncRNAs in text

\begin{tabular}{|c|c|c|c|c|}
\hline LncRNAs & Expression & Functional role & Related factor & Reference \\
\hline IncRNA-CIR & Up & Aggrecan and collagen degradation & MMP-13 and ADAMTS5 & [15] \\
\hline RP11-296A18.3 & Up & Abnormal proliferation of HNPC & miR-138 and HIF1a & [18] \\
\hline PART1 & Up & Influence the expression of ACVR1, E2F3, and VEGFA & miR-34a and miR-148a & [19] \\
\hline HOTAIR & Up & Overexpression of matrix metalloproteinase & $\mathrm{IL}-1 \beta$ & [20] \\
\hline ClLinc01 and CILinc02 & Down & Influence cytokine production & NF-KB & [21] \\
\hline PACER & Up & Influence cytokine production & $\operatorname{cox}-2$ & [22] \\
\hline AC005082.12 & Up & ECM degeneration & EFNA3 & [23] \\
\hline MEG3 & Down & Vascular invasion & VEGF & [24] \\
\hline HCG18 & Up & NP cell apoptosis & miR-146a-5p/TRAF6/NFkB & [25] \\
\hline HOTTIP & Up & Chondrogenic differentiation inhibition & HoxA13 & [26] \\
\hline GAS5 & Up & Apoptosis of chondrocytes & miR-21 & [27] \\
\hline PCGEM1 & Down & Apoptosis of synoviocyte & miR-770 & [28] \\
\hline linc-ADAMTS5 & Down & Aggrecan degradation & ADAMTS5 & [29] \\
\hline TUG1 & Up & NP cell proliferation inhibition & Wnt $\beta$-catenin & {$[30]$} \\
\hline LncRNA-MSR & Up & Overexpression of metalloproteinase & miR-152 & [31] \\
\hline DANCR & Down & Chondrogenic differentiation inhibition & smad3 and STAT3 & [32] \\
\hline
\end{tabular}




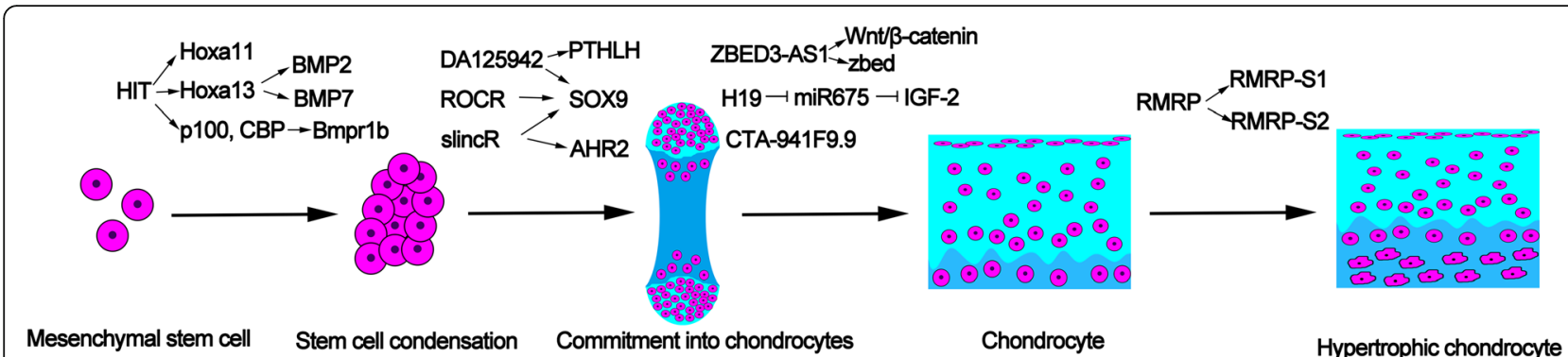

Fig. 1 LncRNA HIT regulates mesenchymal stem cells through LncRNA DA125942, ROCR, and slincR which influence the expression of SOX9 which is important in the early stage of chondrocyte differentiation. LnCRNA ZBED-AS1, H19, and CTA-941F9.9 are involved in the process of chondrocyte differentiation. LncRNA RMRP could promote the chondrocyte differentiating to hypertrophic chondrocytes

GAG production. All the data support that lncRNA ROCR is important for chondrogenesis. LncRNA ROCR in the study was detected from RNA extracted from an aged neck of femur and OA tissue. Further work should confirm the expression of lncRNA ROCR in normal tissue. Morphogenesis gene PTHLH regulates cartilage differentiation and digit condensation and was involved in SOX-9mediated chondrogenesis. CISTR-ACT that encodes a lncRNA termed as DA125942 was found to regulate PTHLH expression in cis and SOX-9 expression in trans. PTHLH and other factors influence the BMP-mediated and SOX9-directed chondrogenesis through balancing a complex signaling network. DA125942 could inhibit the expression of PTHLH and SOX9. Therefore, downregulating the expression of DA125942 may be an approach to promote chondrogenesis [47].

Emerging studies showed that IncRNAs play a part in the stage of chondrocyte progenitor cells into chondrocyte differentiation [48]. H19 is an imprinted maternally gene during fetal development [49]. And H19 may play a role by harboring the miR-675 [50]. Steck and his colleagues [51] have found that the H19-encoded miR-675 modulates collagen type II levels. Insulin growth factor (IGF) is from paternal and is a neighboring gene of H19. H19 influences IGF-2 through sponging miR-675. miR-675 could regulate the expression level of COL2A1 by SOX-9 [52]. In addition, the study revealed that anabolic stimuli upregulated the expression of H19/miR-675 while inflammatory cytokines downregulated them, and their overexpression may be good for cartilage anabolism and tissue degeneration. Meantime, two other lncRNA expression trends of ZBED3-AS1 and CTA-941F9.9 were observed during the chondrogenic differentiation process. Wang et al. [53] demonstrated that the two IncRNAs may function in the early stage of chondrogenic differentiation. Further studies by $\mathrm{Ou}$ et al. [54] found that ZBED3-AS1 could activate the $\mathrm{Wnt} / \beta$-catenin signaling and increased the zbed expression. Overexpression of ZBED3-AS1 upregulates the expression levels of sox 9 and collagen II, but the detailed mechanism requires further investigation.
The impaired cartilage development will cause cartilagehair hypoplasia $(\mathrm{CHH})$. Cartilage-hair hypoplasia is also termed metaphyseal chondrodysplasia. As a result, chondrocytes cannot develop into late phase/hypertrophic chondrocyte [55]. Sox-9 is expressed from the skeletogenetic progenitor cell to cartilage hypertrophy [56]. Mef2c and Runx 2 function in the process from chondrocyte into hypertrophic chondrocyte [57]. Sox9 could be bind to the cis-element of Col2a1 to regulate chondrogenic differentiation while Runx2 and Mef2c regulate the expression of col10a1 during chondrogenic differentiation to the late phase. The mutation of the RMRP gene was reported as the main cause of CHH. RMRP IncRNA and some protein subunits form the small nucleolar ribonucleoprotein particle RNase MRP. RNase MRP is the source of two short RNA designated RMRP-S1 and RMRP-S2 [58]. Mutations in RNase MRP cause human cartilage-hair hypoplasia $(\mathrm{CHH})$. During the course of chondrogenic differentiation, RMRP RNA was found to be involved in the chondrocyte hypertrophy while interfering RMPR RNA will lead to the deregulation of chondrogenic differentiation [59].

\section{Role of IncRNAs in cartilage degeneration}

The degeneration of cartilage could lead to diseases such as osteoarthritis and intervertebral disc degeneration. These diseases induce pain and movement limitations and increase the social burden [60]. Diseases caused by cartilage degeneration were owing to inflammation, oxidative stress, angiogenesis, cell hyperproliferation, ECM degeneration, and chondrocyte apoptosis and autophagy [61-63] (Fig. 2). However, the underlying mechanism was elusive. In recent years, many lncRNAs were found to be correlated with osteoarthritis and intervertebral disc degeneration $[17,18]$. A recent study identified lncRNAs in IDD and spinal cord injury as control with RNA sequencing (RNA-seq). In this study, 1854 lncRNAs were found differentially expressed, of which 1530 lncRNAs could influence 6386 genes through cis-regulatory mechanism [19]. A review described by Li shows that lncRNAs RP11-296A18.3, TUG1, HCG18, MALAT1, SNHG1, 


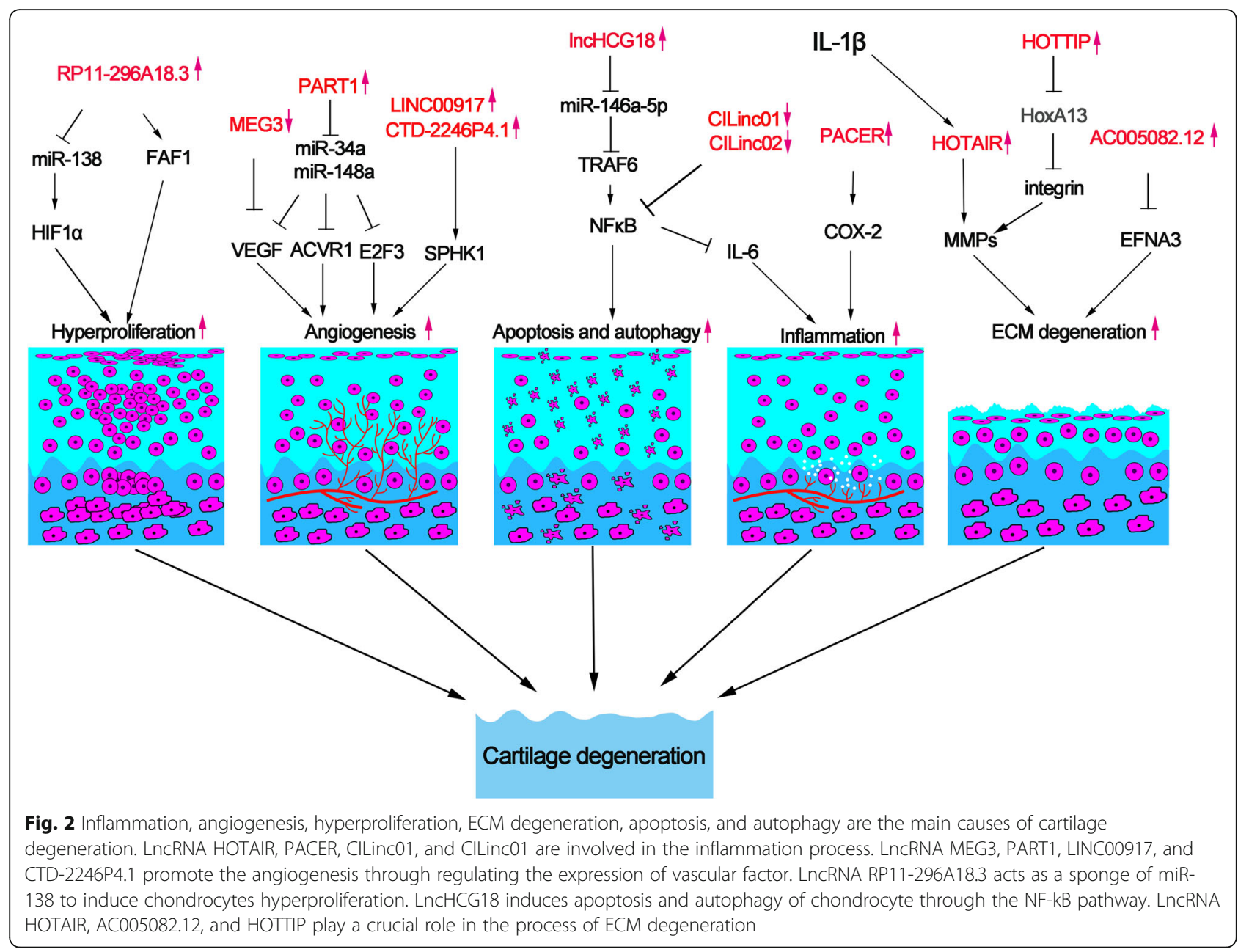

H19, NEAT1, and linc-ADAMTS5 were involved in the IDD process through regulating NP cells [64]. Another study showed that IncRNAs including Gm42770, XLOC-006055, Gm9801, RP23-54G8.4, Gm26848, A530020G20Rik, and Rian comprise the core regulatory network in OA [65].

Inflammation factors such as IL- $1 \beta$ and 6 and TNF play crucial roles in the development of osteoarthritis [66]. IL$1 \beta$ could promote the brain-derived neurotrophic factor (BDNF) and vascular endothelial growth factor (VEGF) which induce angiogenesis [67]. IL-1 $\beta$ was also reported regulating cartilage catabolism, anabolism, and extracellular matrix synthesis [68]. Hox transcript antisense intergenic RNA (HOTAIR) was upregulated about 21-fold in OA compared to the normal cartilage tissue and reported to bind to polycomb repressive complex 2 (PRC2) [69]. Upregulation of IL- $1 \beta$ activated the expression of IncRNA HOTAIR which induced the overexpression of matrix metalloproteinase (MMP) family such as MMP1, MMP3, and MMP9 and chondrocytes apoptosis [20]. In addition, Inflammation factors could induce cartilage degeneration by regulating the expression of lncRNAs. Preculturing with IL-1 $\beta, 125$ lincRNAs were detected differentially expressing in chondrocyte. The lincRNA p50-associated cyclooxygenase 2-extragenic RNA (PACER) and chondrocyte inflammation-associated lincRNAs (CILinc01 and CILinc02) were upregulated to influence the cytokine production, which play a crucial role in the inflammationdriven cartilage degeneration [21]. LincRNA PACER is located upstream to COX-2 locus and regulates COX-2 expression [22]. Silencing CILinc01 and CILinc02 could increase the expression of IL-6 via suppressing NF-kB's activity. And the inhibitor IKK1 of the NF-kB pathway decreased the expression of CILinc01 and CILinc02. In conclusion, CILinc01 and CILinc02 could negatively regulate the inflammation factors to delay cartilage degeneration.

Meantime, angiogenesis is associated with the development of cartilage degeneration [70]. Degeneration of ECM will result in the migration of endothelial cells to cause neovascularization. Intervertebral disc is an avascular and immune-privileged organ [71]. The neovascularization will expose NP to the immune system to cause an immune response, which results in degeneration disease [72]. SPHK1 is a member of SPHK family, which is associated with cell migration and angiogenesis [73]. 
LncRNAs LINC00917 and CTD-2246P4.1 [23] were reported to play a crucial role in the development of IDD through influencing SPHK1 to regulate vascular generation. LncRNA PART1 influences the expression of ACVR1, E2F3, and VEGFA through interacting with has-miR-34a and has-miR-148a [19]. Although RNA-seq data were validated by qRT-PCR, more research should be done to explore the function and mechanism of LncRNA PART1. Maternally expressed gene 3 (MEG3) is located in chromosome 14q32 and acts as an inhibitor in tumor progress by inhibiting angiogenesis [74]. Moreover, lncRNA MEG3 that interacts with SOX2 influences the expression of BMP4 to promote osteogenic differentiation [75]. Meantime, angiogenesis and inflammation are causes of osteoarthritis [76]. LncRNA MEG3 is downregulated and inversely associated with VEGF expression, which causes cartilage remodeling and vascular invasion [24]. These findings suggest that lncRNA MEG3 may play a potential role in cartilage degeneration, although more work should be done to illustrate the underlying mechanism.

Human nucleus pulposus cells (HNPCs), small chondrocyte-like cells, are crucial in the homeostasis of the intervertebral disc. Abnormal proliferation of HNPC will generate cell clusters which cause intervertebral disc degeneration [77]. LncRNA-RP11-296A18.3 promotes the proliferation of HNPCs through sponging miR-138 which inhibits the expression of hypoxia-inducible factor-1 $\alpha$ (HIF1 $\alpha)$ [18]. HIF1 $\alpha$ is an element that could lead to a massive death of HNPCs [78]. On the contrary, another study found that LncRNA-RP11-296A18.3 could promote the expression of Fas-associated protein factor-1 (FAF1), which induced aberrant apoptosis of cartilage cell through the Fas-mediated pathway [79]. Interestingly, LncRNA-RP11-296A18.3 could promote the proliferation of HNPCs but induce apoptosis of cartilage cells in different studies.

Chondrocyte autophagy and apoptosis play a crucial role in the development of cartilage degeneration [80]. Autophagy could remove the generation of reactive oxygen species (ROS) stimulated by a compression stimulus in the nucleus pulposus (NP) cells through sequestrating damaged organelles. In the study, SIRT1 could protect NP cells against apoptosis through promoting autophagy [81]. Interestingly, a recent study reported that the osteogenic differentiation of NP cells was associated with the development of IDD. In the study, Xi et.al found that lncHCG18 could activate the miR-146a-5p/TRAF6/NFkB axis which induced apoptosis and osteogenic differentiation of NP cells and macrophage recruitment [25]. LncHCG was highly expressed in IDD patient, so it may be used as an early diagnostic marker of IDD. Taken together, lncRNAs play a role in cartilage degeneration through inducing apoptosis and autophagy.
ECM serves as the culture medium for the chondrocytes and also serves as the bridge to transfer signals among different chondrocytes [82]. Collagens are the major components of the cartilage structure. Collagen-1 and MMP13 are degeneration ECM markers [83]. Cartilage matrix protein binds to integrin to modulate processes in cartilage development and degeneration [84]. LncRNA CTC523E23.5, RP4-639 J15.1, and RP11-363G2.4 were identified to interact with integrin [23]. So, knowing how to regulate integrin is important to study the cartilage degeneration. Dysregulation of HOX family transcripts may result in limb malformation [85]. HOTTIP, which is known as a regulator of the HoxA gene, was located at the $5^{\prime}$ end of HoxA cluster [86]. In the research studied by Kim et al., HOTTIP was found to regulate integrin by modulating HoxA13 [26]. Moreover, Chen et al. [23] found that lncRNA AC005082.12 interacts with Ephrin-A3 (EFNA3) while MIR132 and RP1138F22.1 interact with Cathepsin L (CTSL) in the development of IDD. Although the sample size is small in the study, the result indicated that lncRNA contributes to cartilage degeneration to some extent.

Other lncRNAs such as lncRNACIR, AC127391.1, AC128677.4, and IGH are also reported being involved in cartilage-related diseases $[15,16]$. These lncRNAs discussed above played certain roles in cartilage development and degeneration and may be the appropriate biomarkers and targets for the treatment of osteoarthritis and intervertebral disc degeneration.

\section{The role of IncRNAs in cartilage regeneration}

Cell, biomaterial, and tissue engineering are the three main approaches for cartilage regeneration. Cell therapies contain transplanting mesenchymal stem cells, autologous chondrocytes, cartilage progenitor cells, and pluripotent stem cells [87]. However, it may take 2-3 years to produce stable and mature ECM after cell transplantation [34]. Biomaterials show simpler regulatory process than cell therapy but do not provide biological function and trigger synthesis of ECM [88, 89]. Tissue engineering combining cells and biomaterials act as a cartilage repair method with unsatisfactory mechanical function [90]. There is still no reliable method to generate articular cartilage to original tissue after injury or disease and no regenerative treatment available for clinical use. Up to now, there is no satisfactory method to cure osteoarthritis and intervertebral disc degeneration.

Emerging evidence showed that lncRNAs are involved in cartilage regeneration (Fig. 3). Targeting lncRNA may be a potential method to OA treatment. For example, silencing of HOTAIR could protect against OA development [20]. Liu et al. reported that 82 lncRNAs were upregulated and 70 were downregulated in OA cartilage compared to normal cartilage [15]. LncRNA PTENP1, 


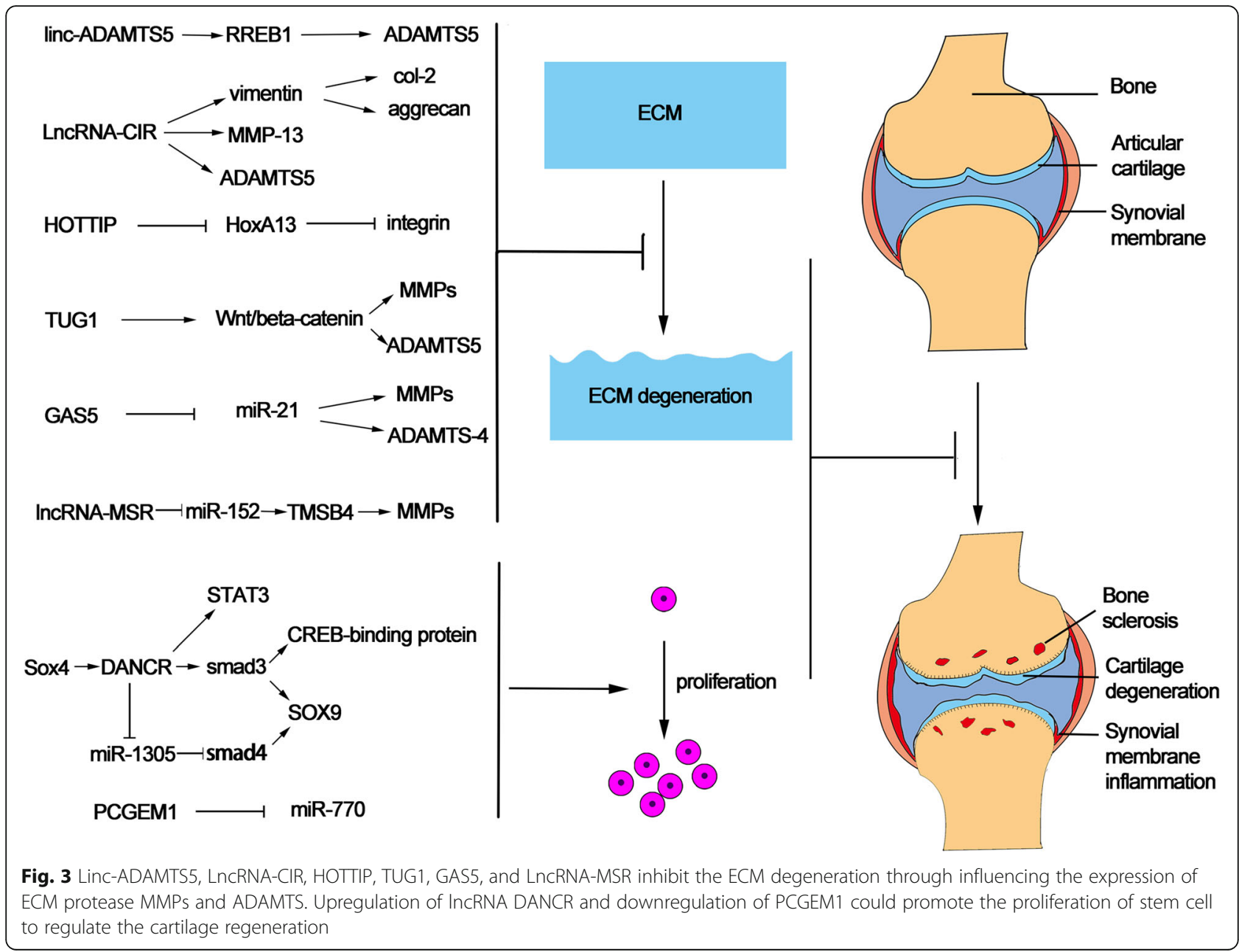

HOTAIR, HOTTIP, UCA1, TUG1, GAS5, TEA, and EGOA were found to be upregulated, while SNHG4, ncR-uPAR, MERI2C, Emx2os, and DISC were found to be downregulated in the OA chondrocytes compared to normal chondrocytes. It may be a suitable approach to silence the IncRNAs upregulated and overexpress the IncRNAs downregulated in OA cartilage. LncRNA-CIR acts as a siRNA to suppress vimentin whose inhibition results in the reduction of the expression of collagen and aggrecan. Furthermore, lncRNA-CIR was demonstrated as a sponge of miR-27b and could regulate the expression of MMP-13. The study revealed that IncRNA-CIR/miR-27/ MMP-13 plays an important role in the degeneration of cartilage ECM [16]. Therefore, silencing lncRNA-CIR may be a potential target for cartilage regeneration. LncRNA growth arrest-specific 5 (GAS5) had been firstly reported as a tumor inhibitor in renal cell carcinoma [91]. GAS5 was also upregulated in osteoarthritis compared to normal tissues [92, 93]. Song and his colleagues [27] found that extraneous GAS5 could upregulate the expressions of MMPs such as MMP-2, MMP-3, MMP-9, MMP-13, and ADAMTS-4. Moreover, LncRNAs could interact with
miRNA response elements (MREs) to affect the expression of mRNAs [94]. GAS5 acted as a negative regulator of miR-21 which induced the apoptosis of chondrocytes and cartilage destruction. Therefore, targeting GAS5 may be developed into a novel therapy to OA once its pathophysiology is completely illustrated. Besides, prostate cancer gene expression marker 1 (PCGEM1) was also a possible target for OA therapy. PCGEM1 sponged miR-770 to inhibit the apoptosis of synoviocyte. Exogenous overexpression of PCGEM1 could induce the proliferation of synoviocyte [28]. Another IncRNA HOTTIP is located within the HoxA cluster and worked by interacting with histone modification complexes [95]. The study by Kim et al. [26] illustrated that the expression of HOTTIP was upregulated in OA chondrocytes with modulating HoxA13 gene level. Knocking down HOTTIP expression, a regulator of HoxA gene, would induce chondrogenic differentiation and suppress cartilage degradation. Collectively, although no effective therapies have yet been discovered to stop OA progression, IncRNA may be a potential choice in the future. 
Targeting certain lncRNA give a new hope to IDD regeneration. ECM degrading enzymes ADAMTS5 serves as a promoter in IDD development $[96,97]$. A newly reported LncRNA named linc-ADAMTS5 is transcribed in the opposite direction to ADAMTS5. Ras-responsive element-binding protein 1 (RREB1) could regulate the expression of ADAMTS5 in NP cells. Linc-ADAMTS5 promotes RREB1 binding to the ADAMTS5 promoter which inhibits the degeneration of ECM [29]. Thus, it was speculated that Linc-ADAMTS5 may play a role in IDD treatment through regulating ECM. Moreover, silencing of lncRNA-CIR was also found to induce the generation of aggrecan and collagen and downregulate the expression of enzymes such as MMP-13 and ADAMTS5 [15]. Chen et al. reported that silencing the expression of lncRNA TUG1 could block the Wnt/betacatenin pathway to promote NP cell proliferation [30]. When NP cells were transfected with TUG1 siRNA, the Wnt/ $\beta$-catenin pathway was greatly inhibited with the reduction of apoptosis, but the cell proliferation was obviously enhanced. Thus, silencing lncRNA TUG1 to promote human NP cell proliferation provides a theoretical basis for the clinical treatment of IDD. LncRNA-MSR is a Tymosin $\beta-4$ (TMSB4) pseudogene, which is a subclass of IncRNA. TMSB4 could inhibit the polymerization of actin filament and induce the expression of metalloproteinase in chondrocytes [92]. LncRNA-MSR acts as a ceRNA through competitively binding to miR-152 to upregulate the TMSB4 expression, which leads to the overexpression of metalloproteinase and disorganization of cytoskeleton and degeneration of ECM [31]. LncRNAMSR may be a potential therapeutic target to decrease the ECM degeneration. The main challenge of these approaches should accomplish with target-specific delivery. Nanoparticle could improve the stability and targetspecific of lncRNAs. Delivery of lncRNA with nanoparticle may be a suitable method to be target-specific and deserves further researches. It is clear that a practical approach to silencing a critical lncRNA candidate may have more broad implications than blocking the initiation or progression of osteoarthritis or intervertebral disc disease. For example, silencing MEG3 intensifies lipopolysaccharide-stimulated damage of human lung cells [98]. Downregulation of lncRNA NEAT1 exerts suppressive effects on immunity [99]. Silencing of lncRNA DGCR5 contributes to the growth, migration, and invasion of cervical cancer [100]. Knockdown of lncRNA ROR suppresses proliferation, migration, and angiogenesis in microvascular endothelial cells [101]. Inhibiting lncRNA GAS5 attenuates damage induced by $\mathrm{H}_{2} \mathrm{O}_{2}$ in retinal ganglion cells [102]. Therefore, if we want to target cartilage-related lncRNAs for cartilage regeneration, more attention should be paid to the side effects of the treatment.
Meantime, promoting the expression of lncRNAs which induce chondrogenesis of stem cells may be an attractive approach to cartilage regeneration. Mesenchymal stem cells are an attractive cell source used in cell therapy for degenerative disease. A considerable amount of literatures have described mesenchymal stem cells have the abilities for osteogenic differentiation, adipogenic differentiation, myogenic differentiation, and chondrogenic differentiation [103]. Stem cell transplanting is one of the methods useful for cartilage regeneration. However, how to effectively induce stem cell differentiation into cartilage remains to be explored. Emerging evidences show that lncRNAs play a crucial role in stem cell differentiation. Nguyen and his colleagues found that 230 lncRNAs and 498 associated miRNAs correlated to chondrogenic differentiation [104]. In a microarray test, lncRNA 50450, 37692, and 16667 were prone to promote chondrogenic differentiation of MSCs [105]. MEG3 regulates chondrogenic differentiation of mesenchymal stem cells by inhibiting TRIB2 expression through methyltransferase EZH2-mediated H3K27me3 [106]. H19 acts as a promoter in cartilage differentiation of ADSCs. However, whether STAT2 and IRF9 are the targets of $\mathrm{H} 19$ and the underlying mechanism remains to be identified in the following works [107]. LncRNA UCA1 were proved to promote chondrogenic differentiation of human bone marrow mesenchymal stem cells through the TGF- $\beta$ pathway. miRNA-145-5p/SMAD5 and miRNA-124-3p/SMAD4 axes were the targets of UCA1 in chondrogenic differentiation [108]. LncRNA DANCR was first studied in hepatocellular carcinoma [109]. It plays an important role in chondrogenic differentiation, which is stimulated by Sox4. A survey conducted by Zhang and his colleagues had shown that Sox4 increased the expression of LncRNA DANCR through binding to it to promote the chondrogenesis [32]. Deletion of DANCR reversed the stimulative effect of Sox 4 on the chondrogenesis of SMSCs. In addition, the STAT3 pathway was involved in the process of chondrogenic differentiation [110]. Moreover, smad3 could activate the expression of SOX9 and recruit CREB-binding protein to promote chondrogenic differentiation [111]. LncRNA DANCR was also found to induce chondrogenic differentiation through upregulating smad3 and STAT3. Furthermore, overexpression of lncRNA DANCR increased the expression of chondrogenic markers and GAG/DNA ratio [112]. Although lncRNAs are shown as a potential target for cartilage treatment, more evidence about lncRNAs' successful treatment in vivo should be investigated. These exciting results suggest that targeting lncRNAs or their related signaling pathways might be a feasible approach for cartilage regeneration. 


\section{Conclusions}

Only a small number of lncRNAs were fully characterized. Microarray is utilized to detect lncRNAs from a known RNA pool, while RNA sequencing (RNA-seq) could detect new lncRNAs [113]. LncRNAs function through various mechanisms in biological processes [114]. For example, lncRNAs could act as RNA decoy, miRNA sponge, competing endogenous RNAs (ceRNA), RNP components, and chromatin modifier's recruitment and modulate translation, splicing, and degeneration of mRNA [115-118]. LncRNAs, genes, proteins, and associated pathways are studied in the co-expression network with informatics approach. Numerous technologies were emerging to study lncRNAs such as RNA immunoprecipitation techniques (RIP-Seq), RNA-Seq, crosslinking, and immunoprecipitation followed by high-throughput sequencing (Clip-Seq), chromatin isolation by RNA purification (ChIRP), and capture hybridization analysis of RNA targets (CHART) [119-123]. After that, a loss-of-function experiment should be carried out to verify the function of lncRNAs. SiRNA and shRNA work well to silence RNA but limiting to the cytoplasmic lncRNA [124]. Then, antisense oligonucleotides (ASOs) begin to be used to silence nuclear lncRNAs [125]. Nowadays, clustered regularly interspaced short palindromic repeats (CRISPR/Cas 9) technology is emerging as an ideal tool to knock out the sequence of certain lncRNA $[126,127]$. One situation we should bear in mind is that CRISPR/Cas 9 is not suitable for knocking out the lncRNAs located in an exon of other genes. In research, once a novel lncRNA was found, we should illustrate the functions of the lncRNAs and verify the functions in vitro and in vivo. In the process of detecting the expression of lncRNAs between different tissues or species, RNA-seq retains the gold standard to identify novel lncRNAs. Second, structure, subcellular location, and binding partner are the three main approaches to predict the function of lncRNAs [33]. LncRNAs do not have the same sequence conservation as protein-coding genes [128]. As a result, the potential function of lncRNAs cannot be predicted by the sequence. Interestingly, the secondary structure of lncRNAs shows more conservation throughout evolution than sequence [129]. And secondary structure shows more importance in the function of lncRNAs. The function of IncRNAs is widespread, so more attention should be paid to the secondary structure of lncRNAs in the future. Chemical and enzymatic probes are utilized to analyze the second and tertiary structure. And the next-generation methods such as Structure-seq, SHAPE-seq, and FragSeq are emerged useful to analyze the structure of lncRNAs [130-132]. Meantime, the location was related to the function of lncRNAs. To some extent, the function of IncRNAs may be indirectly predicted by the function of genes nearby. Single-molecule RNA in situ hybridization is the prominent method to detect the location of lncRNAs. Moreover, hybridizationbased methods are useful to isolate lncRNAs and binding DNA, RNA, and/or protein, which act as a predictor of lncRNA function [133]. In the process of verifying the function of lncRNAs, owing to lncRNAs which show no conservation among species, there are often no homologs of lncRNAs in the animal. It is not easy to find in vivo models to test the function and mechanism of lncRNAs in detail. Nevertheless, many KO animal models for lncRNAs were established with gene disruption, targeted promoter deletions, and premature termination strategies [134136]. CRISPR-Cas9 is a breakthrough genome editing tool. However, lncRNA may retain the function with CRISPRCas9 owing to the lack of ORF $[137,138]$. Therefore, the derivative of Cas9 should be developed to remove the entire gene fragments [139, 140]. LncRNA used as an approach to treat cartilage-related disease is in infancy. As reported by Gogtay, nucleic acid could act as a therapeutic agent [95]. Owing to many lncRNAs involved in the pathological process of cartilage-related diseases, lncRNAtargeting therapy may be a new hope for treatment. RNAi, antisense oligonucleotide, locked nucleic acid GapmeRs, small-molecule inhibitors, and zinc finger nucleases are potential choices to silence the cartilage disease-related lncRNAs [61]. Knocking out the lncRNA which induce cartilage degeneration may be useful. Through advancing technologies, knocking out or overexpressing the key lncRNAs may be potential approaches to treat cartilagerelated diseases.

\section{Abbreviations \\ OA: Osteoarthritis; IDD: Intervertebral disc degeneration; Sox9: Sex- determining region Y (SRY)-box 9; AHR: Aryl hydrocarbon receptor; BDNF: Brain-derived neurotrophic factor; VEGF: Vascular endothelial growth factor; HOTAIR: Hox transcript antisense intergenic RNA; MMP: Matrix metalloproteinase; PACER: p50-associated cyclooxygenase 2-extragenic RNA; HNPC: Human nucleus pulposus cells; HIF1a: Hypoxia-inducible factor-1a; FAF1: Fas-associated protein factor-1; ROS: Reactive oxygen species; ASOs: Antisense oligonucleotides; CRISPR: Clustered regularly interspaced short palindromic repeats}

\section{Acknowledgements}

Not applicable

\section{Authors' contributions}

All authors contributed to the collection, assembly, analysis and interpretation of the data, and critical revision of the article for important intellectual content. All authors read and approved the final version of the manuscript.

\section{Funding}

This research was supported by grants from the National Natural Science Foundation of China (no. 81472504, no. 81401822, and no. 81572177), Science and Technology Foundation of Zhejiang Province (2016C33151), Medical Science and Technology Project of Zhejiang Province of China (2016146428, 2016KYA096, and 2017KY071), and Natural Science Funds of Zhejiang Province (Y17H160033, LQ14H060002, and LY14H060004).

Availability of data and materials Not applicable 


\section{Ethics approval and consent to participate}

Not applicable

\section{Consent for publication}

Not applicable

\section{Competing interests}

The authors declare that they have no competing interests.

\section{Author details}

'Department of Orthopedics, 2nd Affiliated Hospital, School of Medicine, Zhejiang University, \#88 Jie Fang Road, Hangzhou 310009, Zhejiang, People's Republic of China. ${ }^{2}$ Orthopedics Research Institute of Zhejiang University, \#88, Jiefang Road, Hangzhou 310009, China. ${ }^{3}$ Department of Gynecologic Oncology, Women's Hospital, School of Medicine, Zhejiang University, Hangzhou 310009, China.

Received: 6 July 2019 Revised: 17 September 2019 Accepted: 16 October 2019 Published online: 21 November 2019

\section{References}

1. Bressel E, Wing JE, Miller Al, Dolny DG. High-intensity interval training on an aquatic treadmill in adults with osteoarthritis: effect on pain, balance, function, and mobility. J Strength Cond Res. 2014;28:2088-96.

2. Hwang HS, Kim HA. Chondrocyte apoptosis in the pathogenesis of osteoarthritis. Int J Mol Sci. 2015;16:26035-54.

3. Zhang $H$, Zhu T, Zhang L, Wu Q. Stromal cellderived factor1 induces matrix metalloproteinase expression in human endplate chondrocytes, cartilage endplate degradation in explant culture, and the amelioration of nucleus pulposus degeneration in vivo. Int J Mol Med. 2018;41:969-76.

4. Jiang Q, Wang J, Wu X, Ma R, Zhang T, Jin S, et al. LncRNA2Target: a database for differentially expressed genes after IncRNA knockdown or overexpression. Nucleic Acids Res. 2015:43:D193-6.

5. Bar C, Chatterjee S, Thum T. Long noncoding RNAs in cardiovascular pathology, diagnosis, and therapy. Circulation. 2016;134:1484-99.

6. Tye CE, Gordon JA, Martin-Buley LA, Stein JL, Lian JB, Stein GS. Could IncRNAs be the missing links in control of mesenchymal stem cell differentiation? J Cell Physiol. 2015;230:526-34.

7. Brosnan CA, Voinnet O. The long and the short of noncoding RNAs. Curr Opin Cell Biol. 2009;21:416-25.

8. Li YP, Wang Y. Large noncoding RNAs are promising regulators in embryonic stem cells. J Genet Genomics. 2015;42:99-105

9. Boon RA, Jae N, Holdt L, Dimmeler S. Long noncoding RNAs: from clinical genetics to therapeutic targets? J Am Coll Cardiol. 2016;67:1214-26.

10. Briggs JA, Wolvetang EJ, Mattick JS, Rinn JL, Barry G. Mechanisms of long non-coding RNAs in mammalian nervous system development, plasticity, disease, and evolution. Neuron. 2015:88:861-77.

11. Huarte M. The emerging role of IncRNAs in cancer. Nat Med. 2015;21:1253-61.

12. Chen $Y G$, Satpathy AT, Chang HY. Gene regulation in the immune system by long noncoding RNAs. Nat Immunol. 2017;18:962-72.

13. Tang Y, Zhou T, Yu X, Xue Z, Shen N. The role of long non-coding RNAs in rheumatic diseases. Nat Rev Rheumatol. 2017:13:657-69.

14. Fu M, Huang G, Zhang Z, Liu J, Zhang Z, Huang Z, et al. Expression profile of long noncoding RNAs in cartilage from knee osteoarthritis patients. Osteoarthr Cartil. 2015;23:423-32.

15. Liu Q, Zhang X, Dai L, Hu X, Zhu J, Li L, et al. Long noncoding RNA related to cartilage injury promotes chondrocyte extracellular matrix degradation in osteoarthritis. Arthritis Rheumatol. 2014;66:969-78.

16. Li YF, Li SH, Liu Y, Luo YT. Long noncoding RNA CIR promotes chondrocyte extracellular matrix degradation in osteoarthritis by acting as a sponge for Mir-27b. Cell Physiol Biochem. 2017:43:602-10.

17. Wang G, Bu X, Zhang Y, Zhao X, Kong Y, Ma L, et al. LncRNA-UCA1 enhances MMP-13 expression by inhibiting miR-204-5p in human chondrocytes. Oncotarget. 2017;8:91281-90.

18. Wang X, Lv G, Li J, Wang B, Zhang Q, Lu C. LncRNA-RP11-296A18.3/miR138/HIF1A pathway regulates the proliferation ECM synthesis of human nucleus pulposus cells (HNPCs). J Cell Biochem. 2017:118:4862-71.

19. Zhao B, Lu M, Wang D, Li H, He X. Genome-wide identification of long noncoding RNAs in human intervertebral disc degeneration by RNA sequencing. Biomed Res Int. 2016;2016:3684875.
20. Zhang C, Wang P, Jiang P, LV Y, Dong C, Dai $X$, et al. Upregulation of IncRNA HOTAIR contributes to IL-1beta-induced MMP overexpression and chondrocytes apoptosis in temporomandibular joint osteoarthritis. Gene. 2016:586:248-53.

21. Pearson MJ, Philp AM, Heward JA, Roux BT, Walsh DA, Davis ET, et al. Long intergenic noncoding RNAs mediate the human chondrocyte inflammatory response and are differentially expressed in osteoarthritis cartilage. Arthritis Rheumatol. 2016;68:845-56.

22. Krawczyk M, Emerson BM. p50-associated COX-2 extragenic RNA (PACER) activates COX-2 gene expression by occluding repressive NF-kappaB complexes. Elife. 2014;3:e01776.

23. Chen Y, Ni H, Zhao Y, Chen K, Li M, Li C, et al. Potential role of IncRNAs in contributing to pathogenesis of intervertebral disc degeneration based on microarray data. Med Sci Monit. 2015;21:3449-58.

24. Su W, Xie W, Shang Q, Su B. The long noncoding RNA MEG3 is downregulated and inversely associated with VEGF levels in osteoarthritis. Biomed Res Int. 2015;2015:356893.

25. Xi Y, Jiang T, Wang W, Yu J, Wang Y, Wu X, et al. Long non-coding HCG18 promotes intervertebral disc degeneration by sponging miR-146a-5p and regulating TRAF6 expression. Sci Rep. 2017;7:13234.

26. Kim D, Song J, Han J, Kim Y, Chun CH, Jin EJ. Two non-coding RNAs, MicroRNA-101 and HOTTIP contribute cartilage integrity by epigenetic and homeotic regulation of integrin-alpha1. Cell Signal. 2013;25:2878-87.

27. Song J, Ahn C, Chun CH, Jin EJ. A long non-coding RNA, GAS5, plays a critical role in the regulation of miR-21 during osteoarthritis. J Orthop Res. 2014;32:1628-35.

28. Kang Y, Song J, Kim D, Ahn C, Park S, Chun CH, et al. PCGEM1 stimulates proliferation of osteoarthritic synoviocytes by acting as a sponge for miR770. J Orthop Res. 2016;34:412-8.

29. Wang K, Song Y, Liu W, Wu X, Zhang Y, Li S, et al. The noncoding RNA lincADAMTS5 cooperates with RREB1 to protect from intervertebral disc degeneration through inhibiting ADAMTS5 expression. Clin Sci (Lond). 2017; 131:965-79

30. Chen J, Jia YS, Liu GZ, Sun Q, Zhang F, Ma S, et al. Role of LncRNA TUG1 in intervertebral disc degeneration and nucleus pulposus cells via regulating Wnt/beta-catenin signaling pathway. Biochem Biophys Res Commun. 2017; 491:668-74.

31. Liu Q, Hu X, Zhang X, Dai L, Duan X, Zhou C, et al. The TMSB4 pseudogene LncRNA functions as a competing endogenous RNA to promote cartilage degradation in human osteoarthritis. Mol Ther. 2016;24:1726-33.

32. Zhang L, Chen S, Bao N, Yang C, Ti Y, Zhou L, et al. Sox4 enhances chondrogenic differentiation and proliferation of human synovium-derived stem cell via activation of long noncoding RNA DANCR. J Mol Histol. 2015; 46:467-73.

33. Huynh NP, Anderson BA, Guilak F, McAlinden A. Emerging roles for long noncoding RNAs in skeletal biology and disease. Connect Tissue Res. 2017; 58:116-41.

34. Hollander AP, Dickinson SC, Kafienah W. Stem cells and cartilage development: complexities of a simple tissue. Stem Cells. 2010;28:1992-6.

35. Xiao D, Wang R, Hu J, Quan H. Spatial and temporal expression of Smad signaling members during the development of mandibular condylar cartilage. Exp Ther Med. 2017;14:4967-71.

36. Nishimura R, Hata K, Takahata Y, Murakami T, Nakamura E, Yagi H. Regulation of cartilage development and diseases by transcription factors. J Bone Metab. 2017:24:147-53.

37. Lim J, Tu X, Choi K, Akiyama H, Mishina Y, Long F. BMP-Smad4 signaling is required for precartilaginous mesenchymal condensation independent of Sox9 in the mouse. Dev Biol. 2015;400:132-8.

38. Carlson HL, Quinn JJ, Yang YW, Thornburg CK, Chang HY, Stadler HS. LncRNAHIT functions as an epigenetic regulator of chondrogenesis through its recruitment of p100/CBP complexes. PLoS Genet. 2015;11:e1005680.

39. Richards EJ, Zhang G, Li ZP, Permuth-Wey J, Challa S, Li Y, et al. Long noncoding RNAs (LnCRNA) regulated by transforming growth factor (TGF) beta: LncRNA-hit-mediated TGFbeta-induced epithelial to mesenchymal transition in mammary epithelia. J Biol Chem. 2015;290:6857-67.

40. Gross S, Krause Y, Wuelling M, Vortkamp A. Hoxa11 and Hoxd11 regulate chondrocyte differentiation upstream of Runx2 and Shox2 in mice. PLoS One. 2012:7:e43553.

41. Wang P, Li Y, Meng T, Zhang J, Wei Y, Meng Z, et al. KDM6A promotes chondrogenic differentiation of periodontal ligament stem cells by demethylation of SOX9. Cell Prolif. 2017;51:e12413. 
42. Hao N, Whitelaw ML. The emerging roles of AhR in physiology and immunity. Biochem Pharmacol. 2013;86:561-70.

43. Antkiewicz DS, Peterson RE, Heideman W. Blocking expression of AHR2 and ARNT1 in zebrafish larvae protects against cardiac toxicity of 2,3,7,8tetrachlorodibenzo-p-dioxin. Toxicol Sci. 2006;94:175-82.

44. Garcia GR, Goodale BC, Wiley MW, La Du JK, Hendrix DA, Tanguay RL. In vivo characterization of an AHR-dependent long noncoding RNA required for proper Sox9b expression. Mol Pharmacol. 2017;91:609-19.

45. Vance $K W$, Ponting CP. Transcriptional regulatory functions of nuclear long noncoding RNAs. Trends Genet. 2014;30:348-55.

46. Barter MJ, Gomez R, Hyatt S, Cheung K, Skelton A, Xu Y, et al. Long noncoding RNA ROCR contributes to SOX9 expression and chondrogenic differentiation of human mesenchymal stem cells. Development. 2017;144: 4510-21.

47. Maass PG, Rump A, Schulz H, Stricker S, Schulze L, Platzer K, et al. A misplaced IncRNA causes brachydactyly in humans. J Clin Invest. 2012;122:3990-4002.

48. Wang J, Miao J, Meng X, Chen N, Wang Y. Expression of long noncoding RNAs in human bone marrow mesenchymal stem cells cocultured with human amnionderived mesenchymal stem cells. Mol Med Rep. 2017;16:6683-9.

49. Liao J, Yu X, Hu X, Fan J, Wang J, Zhang Z, et al. IncRNA H19 mediates BMP9-induced osteogenic differentiation of mesenchymal stem cells (MSCs) through Notch signaling. Oncotarget. 2017;8:53581-601.

50. Zhao N, Zeng L, Liu Y, Han D, Liu H, Xu J, et al. DLX3 promotes bone marrow mesenchymal stem cell proliferation through H19/miR-675 axis. Clin Sci (Lond). 2017;131:2721-35.

51. Steck E, Boeuf S, Gabler J, Werth N, Schnatzer P, Diederichs S, et al. Regulation of $\mathrm{H} 19$ and its encoded microRNA-675 in osteoarthritis and under anabolic and catabolic in vitro conditions. J Mol Med (Berl). 2012;90: 1185-95.

52. Dudek KA, Lafont JE, Martinez-Sanchez A, Murphy CL. Type II collagen expression is regulated by tissue-specific miR-675 in human articular chondrocytes. J Biol Chem. 2010;285:24381-7.

53. Wang L, Li Z, Li Z, Yu B, Wang Y. Long noncoding RNAs expression signatures in chondrogenic differentiation of human bone marrow mesenchymal stem cells. Biochem Biophys Res Commun. 2015;456:459-64.

54. Ou F, Su K, Sun J, Liao W, Yao Y, Zheng Y, et al. The LncRNA ZBED3-AS1 induces chondrogenesis of human synovial fluid mesenchymal stem cells. Biochem Biophys Res Commun. 2017:487:457-63.

55. Castilla-Cortazar I, Rodriguez De Ita J, Martin-Estal I, Castorena F, Aguirre GA, Garcia de la Garza R, et al. Clinical and molecular diagnosis of a cartilagehair hypoplasia with IGF-1 deficiency. Am J Med Genet A. 2017;173:537-40.

56. Lefebvre V, Dvir-Ginzberg M. SOX9 and the many facets of its regulation in the chondrocyte lineage. Connect Tissue Res. 2017;58:2-14.

57. Kawane T, Komori H, Liu W, Moriishi T, Miyazaki T, Mori M, et al. Dlx5 and mef2 regulate a novel runx2 enhancer for osteoblast-specific expression. J Bone Miner Res. 2014;29:1960-9.

58. Rogler LE, Kosmyna B, Moskowitz D, Bebawee R, Rahimzadeh J, Kutchko K, et al. Small RNAs derived from IncRNA RNase MRP have gene-silencing activity relevant to human cartilage-hair hypoplasia. Hum Mol Genet. 2014;23:368-82.

59. Steinbusch MMF, Caron MMJ, Surtel DAM, Friedrich F, Lausch E, Pruijn GJM, et al. Expression of RMRP RNA is regulated in chondrocyte hypertrophy and determines chondrogenic differentiation. Sci Rep. 2017;7:6440

60. Miotla Zarebska J, Chanalaris A, Driscoll C, Burleigh A, Miller RE, Malfait AM, et al. CCL2 and CCR2 regulate pain-related behaviour and early gene expression in post-traumatic murine osteoarthritis but contribute little to chondropathy. Osteoarthr Cartil. 2017;25:406-12.

61. Chen WK, Yu XH, Yang W, Wang C, He WS, Yan YG, et al. IncRNAs: novel players in intervertebral disc degeneration and osteoarthritis. Cell Prolif. 2017;50:e12313.

62. Hwang MH, Kim KS, Yoo CM, Shin JH, Nam HG, Jeong JS, et al. Photobiomodulation on human annulus fibrosus cells during the intervertebral disk degeneration: extracellular matrix-modifying enzymes. Lasers Med Sci. 2016:31:767-77.

63. Liu Z, Ma C, Shen J, Wang D, Hao J, Hu Z. SDF1/CXCR4 axis induces apoptosis of human degenerative nucleus pulposus cells via the NFkappaB pathway. Mol Med Rep. 2016;14:783-9.

64. Li Z, Li X, Chen C, Li S, Shen J, Tse G, et al. Long non-coding RNAs in nucleus pulposus cell function and intervertebral disc degeneration. Cell Prolif. 2018;51:e12483.

65. Zhang S, An Q, Hu P, Wu X, Pan X, Peng W, et al. Core regulatory RNA molecules identified in articular cartilage stem/progenitor cells during osteoarthritis progression. Epigenomics. 2019;11:669-84.
66. Wehmeyer C, Frank S, Beckmann D, Bottcher M, Cromme C, Konig U, et al. Sclerostin inhibition promotes TNF-dependent inflammatory joint destruction. Sci Transl Med. 2016;8:330ra335.

67. Lee JM, Song JY, Baek M, Jung HY, Kang H, Han IB, et al. Interleukin-1 beta induces angiogenesis and innervation in human intervertebral disc degeneration. J Orthop Res. 2011;29:265-9.

68. Tang Q, Zheng G, Feng Z, Tong M, Xu J, Hu Z, et al. Wogonoside inhibits IL1 beta induced catabolism and hypertrophy in mouse chondrocyte and ameliorates murine osteoarthritis. Oncotarget. 2017:8:61440-56.

69. Li L, Dang Q, Xie H, Yang Z, He D, Liang L, et al. Correction: infiltrating mast cells enhance prostate cancer invasion via altering LncRNA-HOTAIR/PRC2androgen receptor (AR)-MMP9 signals and increased stem/progenitor cell population. Oncotarget. 2016;7:83828.

70. Tabana YM, Al-Suede FS, Ahamed MB, Dahham SS, Hassan LE, Khalilpour S, et al. Cat's whiskers (Orthosiphon stamineus) tea modulates arthritis pathogenesis via the angiogenesis and inflammatory cascade. BMC Complement Altern Med. 2016;16:480.

71. Ma CJ, Liu X, Che L, Liu ZH, Samartzis D, Wang HQ. Stem cell therapies for intervertebral disc degeneration: immune privilege reinforcement by Fas/ FasL regulating machinery. Curr Stem Cell Res Ther. 2015;10:285-95.

72. Sun Z, Zhang M, Zhao XH, Liu ZH, Gao Y, Samartzis D, et al. Immune cascades in human intervertebral disc: the pros and cons. Int J Clin Exp Pathol. 2013;6:1009-14.

73. Shin $\mathrm{KO}$, Seo $\mathrm{CH}$, Cho HH, Oh S, Hong SP, Yoo HS, et al. Ginsenoside compound $\mathrm{K}$ inhibits angiogenesis via regulation of sphingosine kinase- 1 in human umbilical vein endothelial cells. Arch Pharm Res. 2014;37:1183-92.

74. Zhang CY, Yu MS, Li X, Zhang Z, Han CR, Yan B. Overexpression of long non-coding RNA MEG3 suppresses breast cancer cell proliferation, invasion, and angiogenesis through AKT pathway. Tumour Biol. 2017;39: 1010428317701311.

75. Zhuang W, Ge X, Yang S, Huang M, Zhuang W, Chen P, et al. Upregulation of IncRNA MEG3 promotes osteogenic differentiation of mesenchymal stem cells from multiple myeloma patients by targeting BMP4 transcription. Stem Cells. 2015:33:1985-97.

76. Noh KC, Park SH, Yang CJ, Lee GW, Kim MK, Kang YH. Involvement of synovial matrix degradation and angiogenesis in oxidative stress-exposed degenerative rotator cuff tears with osteoarthritis. J Shoulder Elb Surg. 2018; 27:141-50.

77. Tao H, Zhang Y, Wang CF, Zhang C, Wang XM, Wang DL, et al. Biological evaluation of human degenerated nucleus pulposus cells in functionalized self-assembling peptide nanofiber hydrogel scaffold. Tissue Eng Part A. 2014;20:1621-31.

78. Merceron C, Mangiavini L, Robling A, Wilson TL, Giaccia AJ, Shapiro IM, et al. Loss of HIF-1alpha in the notochord results in cell death and complete disappearance of the nucleus pulposus. PLoS One. 2014;9:e110768.

79. Wan ZY, Song F, Sun Z, Chen YF, Zhang WL, Samartzis D, et al. Aberrantly expressed long noncoding RNAs in human intervertebral disc degeneration: a microarray related study. Arthritis Res Ther. 2014;16:465.

80. Meckes JK, Carames B, Olmer M, Kiosses WB, Grogan SP, Lotz MK, et al. Compromised autophagy precedes meniscus degeneration and cartilage damage in mice. Osteoarthr Cartil. 2017;25:1880-9.

81. Ma KG, Shao ZW, Yang SH, Wang J, Wang BC, Xiong LM, et al. Autophagy is activated in compression-induced cell degeneration and is mediated by reactive oxygen species in nucleus pulposus cells exposed to compression. Osteoarthr Cartil. 2013;21:2030-8.

82. Gao Y, Liu S, Huang J, Guo W, Chen J, Zhang L, et al. The ECM-cell interaction of cartilage extracellular matrix on chondrocytes. Biomed Res Int. 2014;2014:648459.

83. Hirata M, Yamaoka T. Effect of stem cell niche elasticity/ECM protein on the self-beating cardiomyocyte differentiation of induced pluripotent stem (iPS) cells at different stages. Acta Biomater. 2018;65:44-52.

84. Zhang $Y$, Wang $\mathrm{H}$. Integrin signalling and function in immune cells. Immunology. 2012;135:268-75.

85. Brison N, Tylzanowski P, Debeer P. Limb skeletal malformations - what the HOX is going on? Eur J Med Genet. 2012;55:1-7.

86. Sasaki YT, Sano M, Kin T, Asai K, Hirose T. Coordinated expression of ncRNAs and HOX mRNAs in the human HOXA locus. Biochem Biophys Res Commun. 2007:357:724-30.

87. Bhattacharjee M, Coburn J, Centola M, Murab S, Barbero A, Kaplan DL, et al. Tissue engineering strategies to study cartilage development, degeneration and regeneration. Adv Drug Deliv Rev. 2015;84:107-22. 
88. Bhardwaj N, Devi D, Mandal BB. Tissue-engineered cartilage: the crossroads of biomaterials, cells and stimulating factors. Macromol Biosci. 2015;15:153-82.

89. Shen Y, Fu Y, Wang J, Li G, Zhang X, Xu Y, et al. Biomaterial and mesenchymal stem cell for articular cartilage reconstruction. Curr Stem Cell Res Ther. 2014;9:254-67.

90. Webber MJ, Khan OF, Sydlik SA, Tang BC, Langer R. A perspective on the clinical translation of scaffolds for tissue engineering. Ann Biomed Eng. 2015:43:641-56

91. Qiao HP, Gao WS, Huo JX, Yang ZS. Long non-coding RNA GAS5 functions as a tumor suppressor in renal cell carcinoma. Asian Pac J Cancer Prev. 2013;14:1077-82.

92. Mirams M, Ayodele BA, Tatarczuch L, Henson FM, Pagel CN, Mackie EJ. Identification of novel osteochondrosis--associated genes. J Orthop Res. 2016;34:404-11.

93. Xing D, Liang JQ, Li Y, Lu J, Jia HB, Xu LY, et al. Identification of long noncoding RNA associated with osteoarthritis in humans. Orthop Surg. 2014:6:288-93.

94. Tay Y, Rinn J, Pandolfi PP. The multilayered complexity of ceRNA crosstalk and competition. Nature. 2014;505:344-52.

95. Pradeepa MM, McKenna F, Taylor GC, Bengani H, Grimes GR, Wood AJ, et al. Psip1/p52 regulates posterior Hoxa genes through activation of IncRNA Hottip. PLoS Genet. 2017;13:e1006677.

96. Ngo K, Pohl P, Wang D, Leme AS, Lee J, Di P, et al. ADAMTS5 deficiency protects mice from chronic tobacco smoking-induced intervertebral disc degeneration. Spine (Phila Pa 1976). 2017;42:1521-8.

97. McCann MR, Veras MA, Yeung C, Lalli G, Patel P, Leitch KM, et al. Wholebody vibration of mice induces progressive degeneration of intervertebral discs associated with increased expression of $11-1$ beta and multiple matrix degrading enzymes. Osteoarthr Cartil. 2017;25:779-89.

98. Li X, Zhang Q, Yang Z. Silence of MEG3 intensifies lipopolysaccharidestimulated damage of human lung cells through modulating miR-4262. Artif Cells Nanomed Biotechnol. 2019:47:2369-78.

99. Chen JX, Xu X, Zhang S. Silence of long noncoding RNA NEAT1 exerts suppressive effects on immunity during sepsis by promoting microRNA125-dependent MCEMP1 downregulation. IUBMB Life. 2019;71:956-68.

100. Liu Y, Chang Y, Lu S, Xiang YY. Downregulation of long noncoding RNA DGCR5 contributes to the proliferation, migration, and invasion of cervical cancer by activating Wnt signaling pathway. J Cell Physiol. 2019;234:11662-9.

101. Qin WW, Xin ZL, Wang HQ, Wang KP, Li XY, Wang X. Inhibiting IncRNA ROR suppresses growth, migration and angiogenesis in microvascular endothelial cells by up-regulating miR-26. Eur Rev Med Pharmacol Sci. 2018;22:7985-93.

102. Miao X, Liang A. Knockdown of long noncoding RNA GAS5 attenuates H2 $\mathrm{O} 2$-induced damage in retinal ganglion cells through upregulating miR124: potential role in traumatic brain injury. J Cell Biochem. 2018.

103. Zhang Y, Chen B, Li D, Zhou X, Chen Z. LncRNA NEAT1/miR-29b-3p/BMP1 axis promotes osteogenic differentiation in human bone marrow-derived mesenchymal stem cells. Pathol Res Pract. 2019;215:525-31.

104. Huynh NPT, Zhang B, Guilak F. High-depth transcriptomic profiling reveals the temporal gene signature of human mesenchymal stem cells during chondrogenesis. FASEB J. 2019;33:358-72.

105. Cao Z, Huang S, Li J, Bai Y, Dou C, Liu C, et al. Long noncoding RNA expression profiles in chondrogenic and hypertrophic differentiation of mouse mesenchymal stem cells. Funct Integr Genomics. 2017:17:739-49.

106. You D, Yang C, Huang J, Gong H, Yan M, Ni J. Long non-coding RNA MEG3 inhibits chondrogenic differentiation of synovium-derived mesenchymal stem cells by epigenetically inhibiting TRIB2 via methyltransferase EZH2. Cell Signal. 2019:63:109379.

107. Pang HL, Zhao QQ, Ma Y, Song YL, Min J, Lu JR, et al. Long noncoding RNA H19 participates in the regulation of adipose-derived stem cells cartilage differentiation. Stem Cells Int. 2019;2019:2139814.

108. Shu T, He L, Wang X, Pang M, Yang B, Feng F, et al. Long noncoding RNA UCA1 promotes chondrogenic differentiation of human bone marrow mesenchymal stem cells via miRNA-145-5p/SMAD5 and miRNA-124-3p/ SMAD4 axis. Biochem Biophys Res Commun. 2019:514:316-22.

109. Yuan SX, Wang J, Yang F, Tao QF, Zhang J, Wang LL, et al. Long noncoding RNA DANCR increases stemness features of hepatocellular carcinoma by derepression of CTNNB1. Hepatology. 2016;63:499-511.

110. Kondo M, Yamaoka K, Sakata K, Sonomoto K, Lin L, Nakano K, et al. Contribution of the interleukin-6/STAT-3 signaling pathway to chondrogenic differentiation of human mesenchymal stem cells. Arthritis Rheumatol. 2015 67:1250-60
111. Furumatsu T, Tsuda M, Taniguchi N, Tajima Y, Asahara H. Smad3 induces chondrogenesis through the activation of SOX9 via CREB-binding protein/ p300 recruitment. J Biol Chem. 2005;280:8343-50.

112. Zhang L, Yang C, Chen S, Wang G, Shi B, Tao X, et al. Long noncoding RNA DANCR is a positive regulator of proliferation and chondrogenic differentiation in human synovium-derived stem cells. DNA Cell Biol. 2017;36:136-42.

113. Bhattarai S, Pontarelli F, Prendergast E, Dharap A. Discovery of novel strokeresponsive IncRNAs in the mouse cortex using genome-wide RNA-seq. Neurobiol Dis. 2017;108:204-12.

114. Marchese FP, Raimondi I, Huarte M. The multidimensional mechanisms of long noncoding RNA function. Genome Biol. 2017;18:206.

115. Hu W, Alvarez-Dominguez JR, Lodish HF. Regulation of mammalian cell differentiation by long non-coding RNAs. EMBO Rep. 2012;13:971-83.

116. Chen R, Jiang T, She Y, Xie S, Zhou S, Li C, et al. Comprehensive analysis of IncRNAs and mRNAs with associated co-expression and ceRNA networks in C2C12 myoblasts and myotubes. Gene. 2018;647:164-73.

117. Niederer RO, Hass EP, Zappulla DC. Long noncoding RNAs in the Yeast $S$. cerevisiae. Adv Exp Med Biol. 2017;1008:119-32.

118. Gonzalez I, Munita R, Agirre E, Dittmer TA, Gysling K, Misteli T, et al. A IncRNA regulates alternative splicing via establishment of a splicing-specific chromatin signature. Nat Struct Mol Biol. 2015:22:370-6.

119. Sun Z, Nair A, Chen X, Prodduturi N, Wang J, Kocher JP. UClncR: ultrafast and comprehensive long non-coding RNA detection from RNA-seq. Sci Rep. 2017;7:14196.

120. Gonzalez-Buendia E, Saldana-Meyer R, Meier K, Recillas-Targa F. Transcriptome-wide identification of in vivo interactions between RNAs and RNA-binding proteins by RIP and PAR-CLIP assays. Methods Mol Biol. 2015; 1288:413-28.

121. Yang YC, Di C, Hu B, Zhou M, Liu Y, Song N, et al. CLIPdb: a CLIP-seg database for protein-RNA interactions. BMC Genomics. 2015:16:51.

122. Vance KW. Mapping long noncoding RNA chromatin occupancy using capture hybridization analysis of RNA targets (CHART). Methods Mol Biol. 2017;1468:39-50.

123. Iyer S, Modali SD, Agarwal SK. Long noncoding RNA MEG3 is an epigenetic determinant of oncogenic signaling in functional pancreatic neuroendocrine tumor cells. Mol Cell Biol. 2017:37:e00278-17.

124. Chen S, Wu DD, Sang XB, Wang LL, Zong ZH, Sun KX, et al. The IncRNA HULC functions as an oncogene by targeting ATG7 and ITGB1 in epithelial ovarian carcinoma. Cell Death Dis. 2017;8:e3118.

125. Lennox KA, Behlke MA. Cellular localization of long non-coding RNAs affects silencing by RNAi more than by antisense oligonucleotides. Nucleic Acids Res. 2016;44:863-77.

126. Doetschman T, Georgieva T. Gene editing with CRISPR/Cas9 RNA-directed nuclease. Circ Res. 2017;120:876-94.

127. Joung J, Engreitz JM, Konermann S, Abudayyeh OO, Verdine VK, Aguet F, et al. Genome-scale activation screen identifies a IncRNA locus regulating a gene neighbourhood. Nature. 2017;548:343-6.

128. Breschi A, Gingeras TR, Guigo R. Comparative transcriptomics in human and mouse. Nat Rev Genet. 2017:18:425-40.

129. Johnsson P, Lipovich L, Grander D, Morris KV. Evolutionary conservation of long non-coding RNAs; sequence, structure, function. Biochim Biophys Acta. 1840;2014:1063-71.

130. Fang R, Moss WN, Rutenberg-Schoenberg M, Simon MD. Probing Xist RNA structure in cells using Targeted Structure-Seq. PLoS Genet. 2015;11: e1005668.

131. Loughrey D, Watters KE, Settle AH, Lucks JB. SHAPE-Seq 2.0: systematic optimization and extension of high-throughput chemical probing of RNA secondary structure with next generation sequencing. Nucleic Acids Res. 2014;42.

132. Underwood JG, Uzilov AV, Katzman S, Onodera CS, Mainzer JE, Mathews $\mathrm{DH}$, et al. FragSeq: transcriptome-wide RNA structure probing using highthroughput sequencing. Nat Methods. 2010;7:995-1001.

133. McHugh CA, Russell P, Guttman M. Methods for comprehensive experimental identification of RNA-protein interactions. Genome Biol. 2014; 15:203.

134. Li L, Chang HY. Physiological roles of long noncoding RNAs: insight from knockout mice. Trends Cell Biol. 2014;24:594-602.

135. Rapicavoli NA, Poth EM, Blackshaw S. The long noncoding RNA RNCR2 directs mouse retinal cell specification. BMC Dev Biol. 2010;10:49.

136. Mancini-Dinardo D, Steele SJ, Levorse JM, Ingram RS, Tilghman SM. Elongation of the Kcnq1 ot1 transcript is required for genomic imprinting of neighboring genes. Genes Dev. 2006;20:1268-82. 
137. Necsulea A, Soumillon M, Warnefors M, Liechti A, Daish T, Zeller U, et al. The evolution of IncRNA repertoires and expression patterns in tetrapods. Nature. 2014;505:635-40.

138. Cabili MN, Trapnell C, Goff L, Koziol M, Tazon-Vega B, Regev A, et al. Integrative annotation of human large intergenic noncoding RNAs reveals global properties and specific subclasses. Genes Dev. 2011;25:1915-27.

139. Hilton IB, D'I ppolito AM, Vockley CM, Thakore PI, Crawford GE, Reddy TE, et al. Epigenome editing by a CRISPR-Cas9-based acetyltransferase activates genes from promoters and enhancers. Nat Biotechnol. 2015;33:510-7.

140. Konermann S, Brigham MD, Trevino AE, Joung J, Abudayyeh OO, Barcena C, et al. Genome-scale transcriptional activation by an engineered CRISPR-Cas9 complex. Nature. 2015;517:583-8.

\section{Publisher's Note}

Springer Nature remains neutral with regard to jurisdictional claims in published maps and institutional affiliations. 